\title{
Absence of Hikeshi, a nuclear transporter for heat-shock protein HSP70, causes infantile hypomyelinating leukoencephalopathy
}

\author{
Catalina Vasilescu ${ }^{1}$, Pirjo Isohanni ${ }^{1,2}$, Maarit Palomäki ${ }^{3}$, Helena Pihko², Anu Suomalainen ${ }^{\star, 1,4,5}$ \\ and Christopher J Carroll ${ }^{\star, 1}$
}

Genetic leukoencephalopathies are a heterogeneous group of central nervous system disorders with white matter involvement. In a Finnish patient, we identified a novel homozygous disease-causing variant in $H I K E S H I, c .11 G>C$, p.(Cys4Ser), leading to hypomyelinating leukoencephalopathy with periventricular cysts and vermian atrophy. A founder Ashkenazi-Jewish diseasecausing variant recently linked Hikeshi and its heat-shock protective function to leukoencephalopathy. In our patient, clinical features of lower limb spasticity, optic atrophy, nystagmus, and severe developmental delay were similar to reported patients. Additional features included vermian atrophy, epileptic seizures, and an ovarian tumor. Structural modeling and protein analyses revealed that modified interactions inside Hikeshi's hydrophobic pockets induce protein instability. The patient's cells showed impaired nuclear translocation of HSP70 during heat shock, and decreased ERO1-L $\alpha$, an endoplasmic reticulum (ER) oxidoreductase. Overall, we show that: (1) the clinical spectrum associated with Hikeshi deficiency extends to leukoencephalopathy with vermian atrophy and epilepsy; (2) the cellular disease process involves both nuclear chaperone and ER functions.

European Journal of Human Genetics (2017) 25, 366-370; doi:10.1038/ejhg.2016.189; published online 21 December 2016

\section{INTRODUCTION}

Leukoencephalopathies are central nervous system disorders with significant white matter involvement ${ }^{1}$ and heterogeneous genetic background. ${ }^{2}$ Hikeshi emerged recently as a novel genetic cause for leukoencephalopathy with acquired microcephaly in the AshkenaziJewish (AJ) population. ${ }^{3}$ Hikeshi transports heat-shock protein 70 (HSP70) into the nucleus upon its activation, and thereby contributes to cellular stress responses. ${ }^{4,5}$ Here, we report the genetic and functional characteristics of a new recessive Hikeshi disease-causing variant, found in a Finnish patient with leukoencephalopathy, spastic paraparesis, cerebellar ataxia, and epilepsy.

\footnotetext{
MATERIALS AND METHODS

Whole-exome sequencing (WES) was carried out using the NimbleGen SeqCap EZ Exome v2.0 kit (Roche, Pleasanton, CA, USA) and the data processed with the pipeline of the Finnish Institute for Molecular Medicine. ${ }^{6}$ The HIKESHI variant was submitted to LOVD database (individual ID 00081825; http:// databases.lovd.nl/shared/variants/0000134729). We modeled the effects of the identified variant and of the reported $\mathrm{AJ}$ variant using the software Discovery Studio 4.5 (Biovia, San Diego, CA, USA) and the crystal structure of human Hikeshi $^{7}$ as template (PDB id 3WVZ). Supplementary Methods contain details on Sanger sequencing, protein analyses, heat shock, and tunicamycin treatment.
}

\section{RESULTS}

Our patient, a female, was the first child of a consanguineous healthy Finnish couple with two healthy sons (Figure 1a). Table 1 summarizes her clinical features and of reported AJ patients. She was born at term, but was small for gestational age with birth weight of $2330 \mathrm{~g}$ $(-1.8 \mathrm{SD})$, height of $47 \mathrm{~cm}(-2.2 \mathrm{SD})$, head circumference of $32.5 \mathrm{~cm}(-2 \mathrm{SD})$, and Apgar scores of 9/9. She was irritable and had increased muscle tone from birth, and her motor development was delayed. Spasticity of the lower limbs, ataxia, athetosis, and nystagmus were diagnosed by 1 year of age. At the age of 2 years, she was able to say a few words and take some steps supported, but never learned to walk independently.

At 2 years and 3 months, during a febrile infection, she had an encephalitis-like episode with prolonged seizures, unconsciousness, and nuchal rigidity. EEG revealed bilateral decreased activity with spikes in the right temporal lobe, CT scan showed right-sided temporoparietal diffuse hyperdensity, and CSF analysis showed mild leukocytosis, but no bacterial, viral, or antibody findings. She was treated in an intensive care unit for 4 days, and received antiepileptic, antibiotic, and antiviral treatment. Her condition deteriorated: she was no longer able to sit or crawl. She developed epilepsy with psychomotor, tonic-clonic, and absence seizures, and was on continuous antiepileptic medication, but did not became

${ }^{1}$ Research Programs Unit, Molecular Neurology, Biomedicum-Helsinki, University of Helsinki, Helsinki, Finland; ${ }^{2}$ Department of Pediatric Neurology, Children's Hospital, University of Helsinki and Helsinki University Hospital, Helsinki, Finland; ${ }^{3}$ Department of Radiology, University of Helsinki and Helsinki University Hospital, Helsinki, Finland; ${ }^{4}$ Department of Neurology, Helsinki University Hospital and Clinical Neurosciences, University of Helsinki, Helsinki, Finland; ${ }^{5}$ Neuroscience Center, University of Helsinki, Helsinki, Finland

*Correspondence: Professor A Suomalainen, Research Programs Unit, Molecular Neurology, Biomedicum-Helsinki, r.C523b, Haartmaninkatu 8, 00290 Helsinki, Finland. Tel: +358 947171 959; Fax: +358 947171 964; E-mail: anu.wartiovaara@helsinki.fi

or Dr CJ Carroll, Research Programs Unit, Molecular Neurology, Biomedicum-Helsinki, University of Helsinki, r.C518b, Haartmaninkatu 8, 00290 Helsinki, Finland. Tel: +358 947171 892; Fax: +358 947171 964; E-mail: christopher.carroll@helsinki.fi

Received 18 August 2016; revised 2 November 2016; accepted 22 November 2016; published online 21 December 2016 
totally seizure-free. At 6 years of age, she was diagnosed with a malignant ovarian endodermal sinus tumor, which was treated with chemotherapy.

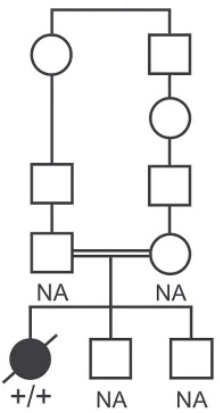

c. $11 \mathrm{G}>\mathrm{C} ;$ p.(Cys4Ser)

e

c. $11 \mathrm{G}>\mathrm{C} \quad$ c. $160 \mathrm{G}>\mathrm{C}$

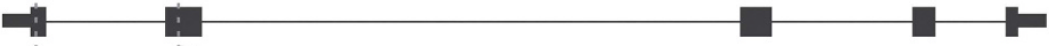

:p.(Cys4Ser) ${ }^{\cdots}$, p.(Val54Leu)

$\mathrm{NH} 2$

$1^{1 \cdots}$

Bos taurus

Mus musculus

Gallus gallus

Xenopus tropict

Caenorhabditis elegans

Drosophila melanogaster

Anopheles gambiae

Arabidopsis thalian

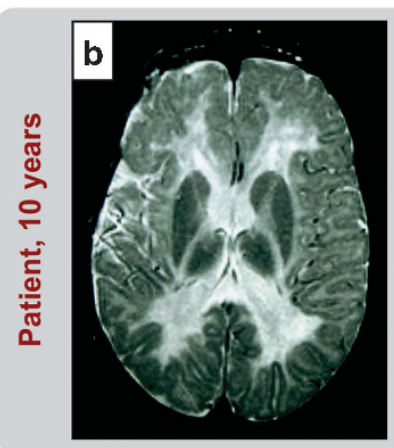

HIKESHI

$-\mathrm{COOH}$

197

$\begin{array}{lll}48 & \text { EGMGGSVYFSYPD } & 60 \\ 48 & \text { EGMGGSVYFSYPD } & 60 \\ 48 & \text { EGMGGSVYFSYPD } & 60 \\ 48 & \text { EGMGGSVYFCYPD } & 60 \\ 48 & \text { ERMGGSVYFSYPD } & 60 \\ 48 & \text { EGLGGAVYLCVPG } & 60 \\ 50 & \text { DGIGGSVYIRWPT } & 62 \\ 50 & \text { VGTSAAIYFSWPD } & 62 \\ 51 & \text { DGMAGGVYFSWPD } & 63 \\ 48 & \text { VGMGAKVYFQWPG } & 60 \\ 56 & \text { PDKALAVYVQSPG } & 68\end{array}$

MLNALGVIVSGRL

---MFGAICAGRL 10
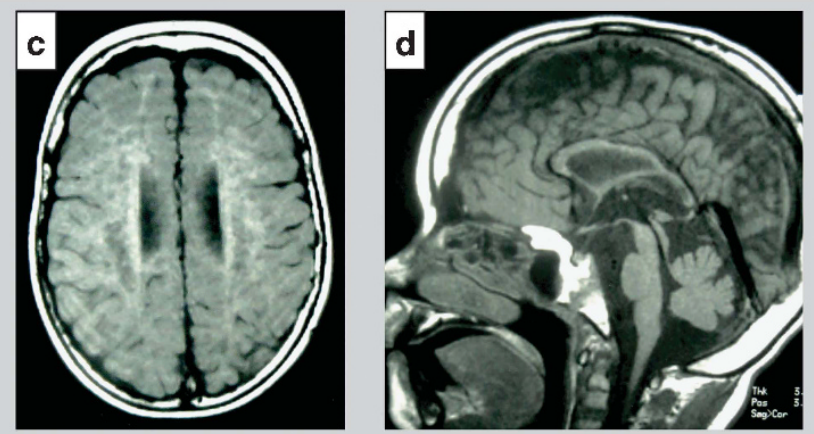

f

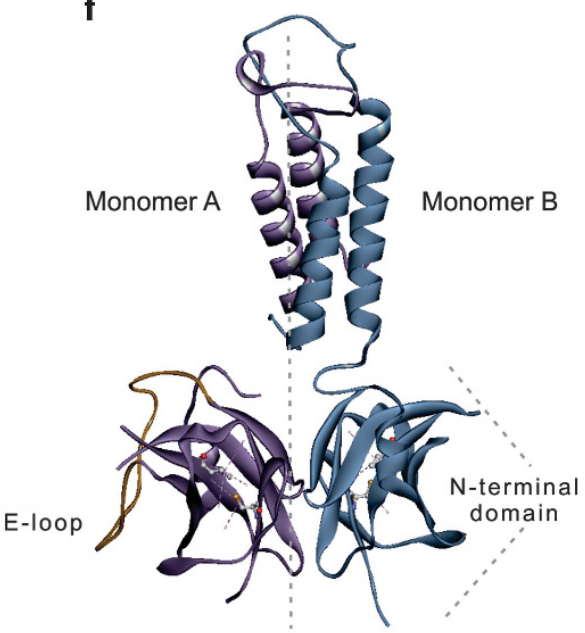

h

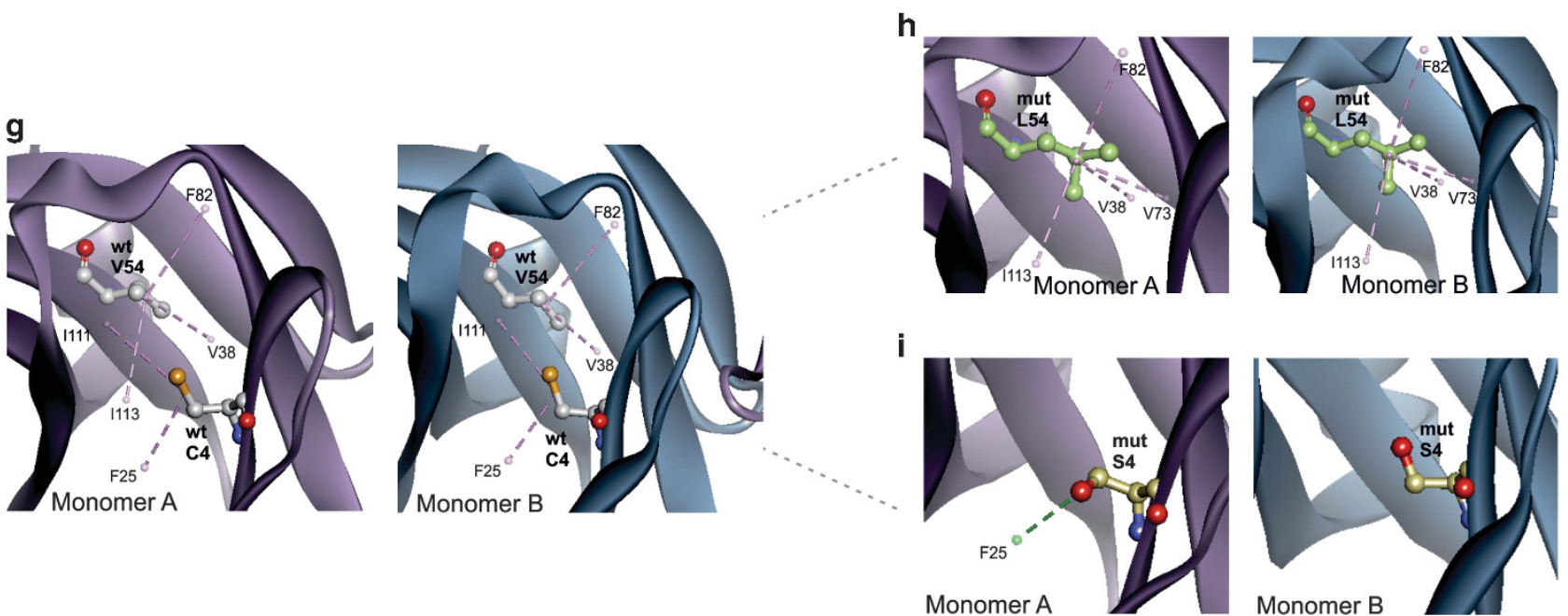

g
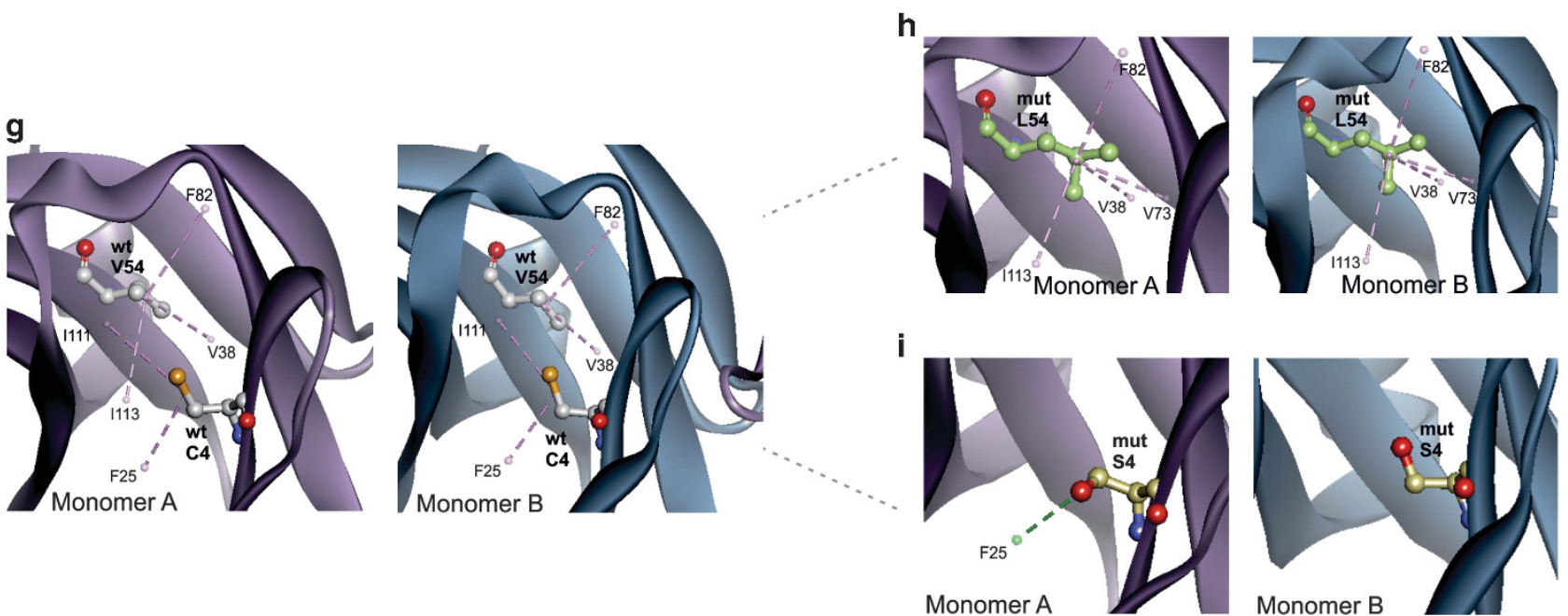

Her disease progressed and her skills regressed. She was no longer able to propel her wheelchair or move independently, but she communicated with figures. She developed severe spasticity, ataxia,

Figure 1 Pedigree, patient's brain MRI at 10 years of age, and consequences of disease-causing variants at protein level. (a) Pedigree of the family: the patient is indicated by filled symbol. (b) Axial view of T2-weighted MR image shows diffuse hypomyelination and (c) axial view of T1-weighted MR image shows cystic changes in periventricular white matter. (d) Sagittal view of T1-weighted MR image shows vermian atrophy. (e) The variant p.(Cys4Ser) found in our patient and $\mathrm{p}$.(Val54Leu) reported in AJ patients reside within N-terminal domain and are conserved in vertebrates. (f) Overview of the asymmetric homodimer arrangement of Hikeshi showing the affected amino acids within NTDs, based on human Hikeshi crystal structure (PDB id 3WVZ). (g) Interactions established by the side chains of wild-type amino acids Cys4 and Val54 within the hydrophobic pocket of monomer A and B. (h) Bonding pattern for p.(Val54Leu) variant: increased number of hydrophobic interactions and loss of wild-type asymmetry of bonding. (i) Interactions induced by p.(Cys4Ser) variant: hydrophobic interactions are lost; Ser4 in monomer A forms a hydrogen bond. Functional domains of Hikeshi: NTD, N-terminal domain; E-loop, extended loop; CTD, C-terminal domain; FLR, flexible linker region. Violet dash lines, hydrophobic bonds. Green dash line, hydrogen bond. 


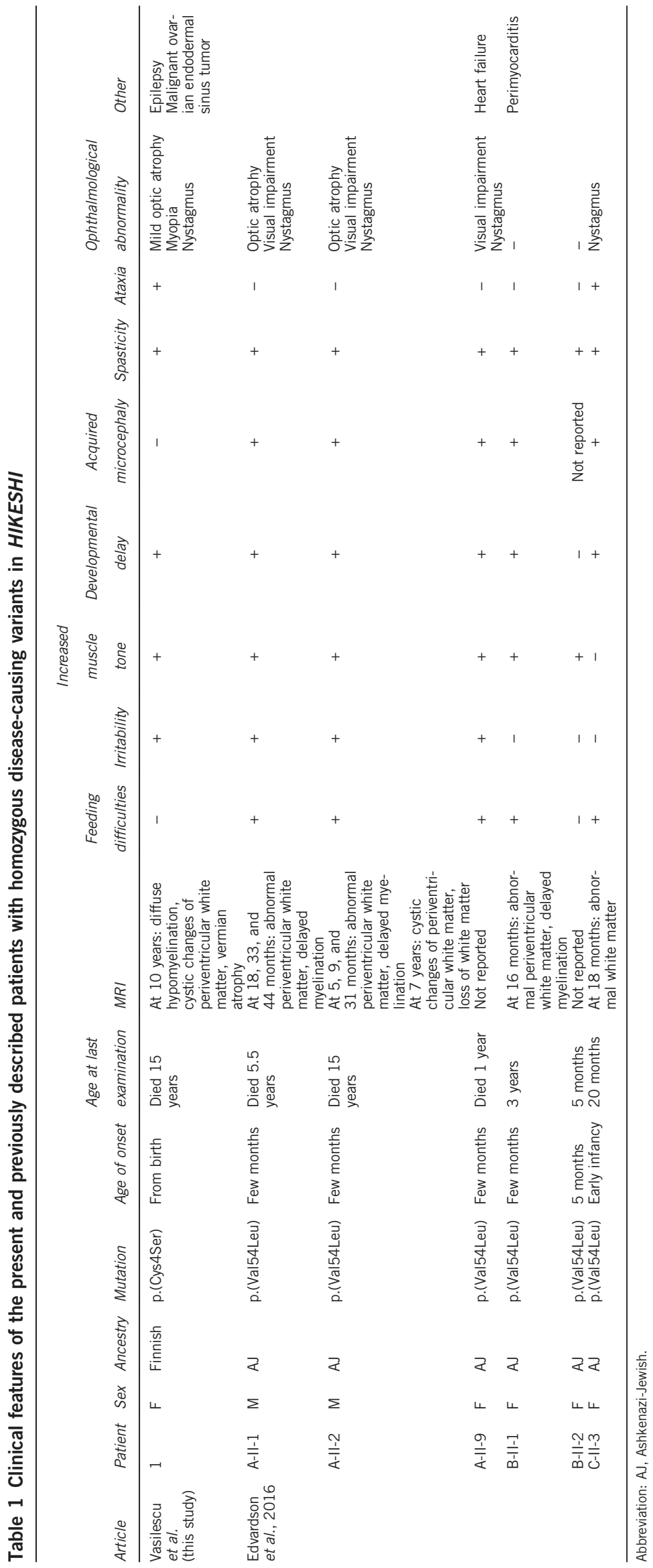


nystagmus, mild optic atrophy, and myopia. Brain CT and MRI showed leukoencephalopathy with periventricular cysts and vermian atrophy (Figures 1b-d). Blood lactate was slightly elevated (1.5-3.4 mmol/l, reference range $0.5-2.2 \mathrm{mmol} / \mathrm{l}$ ), muscle histology was normal, but respiratory chain enzyme analysis showed partial complex I+III deficiency in the muscle (at 10 years of age) (Supplementary Table 1). Other laboratory evaluations, including pyruvate, thyroid hormones, liver transaminases, alpha fetoprotein, urine organic acids, and karyotyping were unremarkable. She died of pneumonia at 15 years of age.

Variant prioritization of the patient's WES data led to identification of a homozygous missense change (Supplementary Figures $1 \mathrm{a}$ and b): HIKESHI (NM_016401.3; NP_057485.2), hg19 chr11:g.86013501G > C, c.11G $>$ C, p.(Cys4Ser). The variant was confirmed by Sanger sequencing (Supplementary Figure 1c); parental samples were unavailable. The variant was absent in ExAC (http://exac.broadinstitute.org/), whereas SISu database (http://www.sisuproject.fi/) contained one heterozygous carrier among 7142 Finnish exomes $(\mathrm{MAF}=0.00014) . \mathrm{SIFT}^{8}$ rated the variant as deleterious (0.04) and the $\mathrm{CADD}^{9} \mathrm{C}$-score was 24.3. The amino acid site altered in our patient, Cys4, is conserved across vertebrates (Figure 1e).

The functional Hikeshi is an asymmetric homodimer, and both our patient's and AJ variants are located within the $\mathrm{N}$-terminal domain (Figure 1f) that folds into a hydrophobic pocket involved in nuclear pore recognition.

Modeling of the p.(Cys4Ser) variant predicted the loss of hydrophobic interactions established by wild-type Cys4 in both monomers (Figures $1 \mathrm{~g}$ and $\mathrm{i}$ ). Immunoblotting showed undetectable levels of Hikeshicys4Ser in patient fibroblasts (Figure 2a). The model of AJ mutation p.(Val54Leu) showed enhanced hydrophobic interactions and loss of bonding asymmetry between the two monomers characteristic for wild-type Val54 (Figures $1 \mathrm{~g}$ and h).

In response to heat shock, HSP70 translocates from cytoplasm into nucleus to protect nuclear structures from damage. After $1 \mathrm{~h}$ heat shock at $42{ }^{\circ} \mathrm{C}$, control fibroblasts exhibited a strong punctate nuclear accumulation of $\mathrm{HSC} / \mathrm{HSP} 70$ (constitutive and inducible forms, respectively), whereas HikeshiCys4Ser fibroblasts showed a weak accumulation (Figure 2c). Both control and patient fibroblasts showed phosphorylation of HSF1-S326 (Figure 2b), indicating the activation of heat-shock response.

The heat-shock and endoplasmic reticulum (ER) stress responses have common mediators, prompting us to screen the ER stress markers. HikeshiCys4Ser fibroblasts showed normal levels of unfolded protein response (UPR) markers IRE1 $\alpha$ and BiP, whereas ERO1-L $\alpha$, an ER oxidoreductase, was clearly reduced (Figures $2 \mathrm{~d}$ and e). Fibroblasts were then immortalized for further experiments. A side effect of the immortalization was increased BiP, but ERO1-L $\alpha$ remained low. When challenged with the ER stressor tunicamycin, patient cells responded normally by upregulating IRE1 $\alpha$ and $\mathrm{BiP}$ (Figure 2f). The reduction of ERO1-L $\alpha$ in our patient cells is therefore independent of a generalized activation of ER-UPR or defective ER-UPR expression.

\section{DISCUSSION}

We describe here a Finnish patient with a progressive neurodegenerative disorder, prominent leukoencephalopathy, and a new homozygous disease-causing variant in Hikeshi. The clinical features of severe developmental delay, spasticity, nystagmus, and optic atrophy were similar to the reported patients of AJ ancestry. ${ }^{3}$ In addition, our patient presented vermian atrophy, epileptic seizures, and ovarian

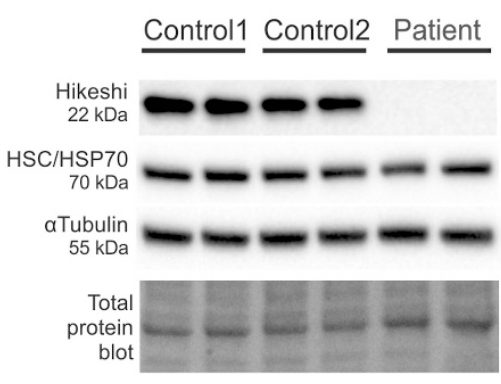

b

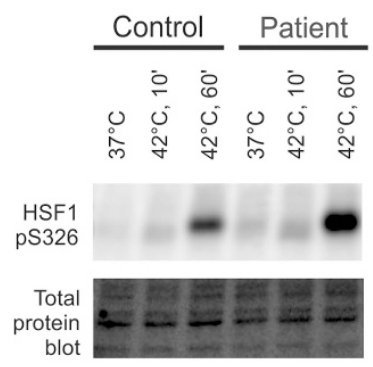

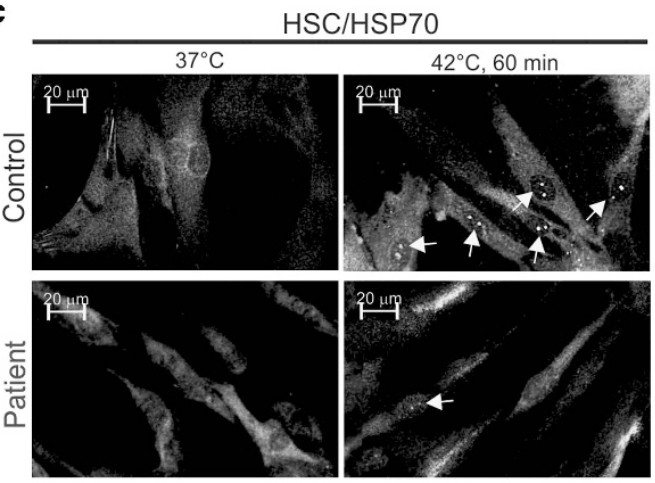

$\mathbf{f}$

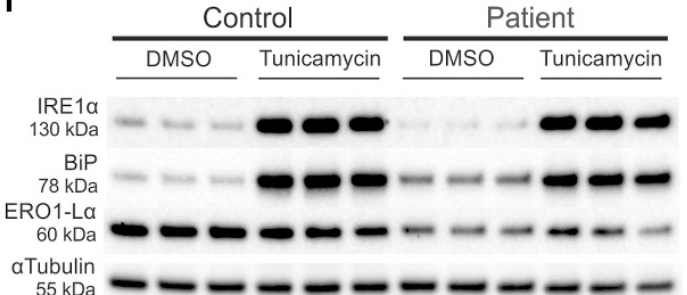

Figure 2 Loss of Hikeshi has consequences in the nucleus and endoplasmic reticulum (ER). (a) Hikeshi is undetectable in patient's fibroblasts harboring p.(Cys4Ser) change. HSC/HSP70 levels are normal in patient's primary fibroblasts. (b) Patient's immortalized fibroblasts are able to induce the heat-shock response by phosphorylating HSF1-S326. (c) Indirect immunostaining of HSC/HSP70 in control and patient's immortalized fibroblasts, in normal conditions and in heat shock. Strong nuclear punctate accumulations appear in control cells, whereas in patient's fibroblasts, the staining is weaker. (d) Screening of ER stress markers indicates a reduction of ERO1-L $\alpha$ in patient's primary fibroblasts. (e) Relative quantification of Western blot ER stress markers. The error bars show normalized data range between the two controls. (f) Patient's immortalized fibroblasts respond to tunicamycin-induced ER stress in a similar way to control fibroblasts. 
cancer, which as a single finding may be coincidental, albeit cellular stress responses mitigated by heat-shock proteins have been found in both neurodegenerative disorders and cancer. ${ }^{10,11}$ At 2 years of age, our patient had severe deterioration following a febrile illness, with suspected encephalitis. It is noteworthy that three out of six AJ patients died suddenly during short febrile illnesses, implying abnormal responses to heat stress. ${ }^{3}$

Our structural modeling and immunoblotting showed that altered interactions inside Hikeshi hydrophobic pockets induce protein instability. Similar to Hikeshival54Leu, we observed a decreased heatinduced nuclear translocation of HSP70 in HikeshiCys4Ser fibroblasts, supporting the idea that a defective response to heat stress is a possible mechanism for Hikeshi disorders. ${ }^{3}$

ER-UPR has been described in several myelin disorders, including Pelizaeus-Merzbacher disease and vanishing white matter disease. ${ }^{12}$ Our patient's fibroblasts presented normal levels of the ER-UPR markers IRE1 $\alpha$ and BiP, which were also normally upregulated upon tunicamycin treatment. However, we found a clear reduction of ERO1-L $\alpha$, an ER oxidoreductase involved in disulfide bond formation and ER redox state, suggesting that ER may contribute to the cellular pathology of Hikeshi disorders.

Overall, our patient indicates that Hikeshi deficiency results in a complex neurodegenerative phenotype involving white matter and cerebellum, sometimes progressing to epilepsy. In diagnostic settings, HIKESHI screening should be considered in hypomyelinating leukoencephalopathies, especially if the disease progresses during febrile illnesses.

The causative link established between neurodegenerative disorders and chaperones/co-chaperones argues for a critical role of rigorous protein quality control in the central nervous system. ${ }^{13}$ Some examples include HSPD1 causing hypomyelinating leukoencephalopathy ${ }^{14}$ and hereditary spastic paraplegia $13,{ }^{15}$ DNAJC6 and DNAJC13 involved in Parkinson's disease, ${ }^{16,17}$ and DNAJC19 involved in dilated cardiomyopathy with ataxia. ${ }^{18,19}$

Hikeshi, a binding partner and nuclear transporter of HSP70, is a new addition to this group and highlights the essential role of adequate stress responses, including heat-shock response, for normal development and maintenance of the nervous system.

\section{CONFLICT OF INTEREST}

The authors declare no conflict of interest.

\section{ACKNOWLEDGEMENTS}

We thank the patient's family for participation in our study; Anu Harju for technical assistance; Virginia Brilhante, Eino Palin, and Simo Ojanen for bioinformatic support; Wendy Chung and Claudia Gonzaga-Jauregui for comments. We thank funding resources: University of Helsinki (CJC and AS), Academy of Finland (AS), European Research Council (AS), Sigrid Jusélius Foundation (AS), Jane and Aatos Erkko Foundation (AS), United Mitochondrial Disease Foundation (AS), and Helsinki Doctoral Programme in Biomedicine $(\mathrm{CV})$.

1 Vanderver A, Prust M, Tonduti D et al: Case definition and classification of leukodystrophies and leukoencephalopathies. Mol Genet Metab 2015; 114: 494-500.

2 Parikh S, Bernard G, Leventer RJ et al: A clinical approach to the diagnosis of patients with leukodystrophies and genetic leukoencephelopathies. Mol Genet Metab 2015; 114: 501-515.

3 Edvardson S, Kose S, Jalas C et al: Leukoencephalopathy and early death associated with an Ashkenazi-Jewish founder mutation in the Hikeshi gene. J Med Genet 2016; 53: 132-137.

4 Kose S, Furuta M, Imamoto N: Hikeshi, a nuclear import carrier for Hsp70s, protects cells from heat shock-induced nuclear damage. Cell 2012; 149: 578-589.

5 Kose S, Imamoto N: Nucleocytoplasmic transport under stress conditions and its role in HSP70 chaperone systems. Biochim Biophys Acta 2014; 1840: 2953-2960.

6 Sulonen AM, Ellonen P, Almusa $\mathrm{H}$ et al: Comparison of solution-based exome capture methods for next generation sequencing. Genome Biol 2011; 12: R94.

7 Song J, Kose S, Watanabe A et al: Structural and functional analysis of Hikeshi, a new nuclear transport receptor of Hsp70s. Acta Crystallogr D Biol Crystallogr 2015; 71 : 473-483.

8 Kumar P, Henikoff S, Ng PC: Predicting the effects of coding non-synonymous variants on protein function using the SIFT algorithm. Nat Protoc 2009; 4: 1073-1082.

9 Kircher M, Witten DM, Jain P, O'Roak BJ, Cooper GM, Shendure J: A general framework for estimating the relative pathogenicity of human genetic variants. Nat Genet 2014; 46: 310 .

10 Sherman MY, Gabai VL: Hsp70 in cancer: back to the future. Oncogene 2015; 34: 4153-4161.

11 Lindberg I, Shorter J, Wiseman RL, Chiti F, Dickey CA, McLean PJ: Chaperones in neurodegeneration. J Neurosci 2015; 35: 13853-13859.

12 Lin W, Popko B: Endoplasmic reticulum stress in disorders of myelinating cells. Nat Neurosci 2009; 12: 379-385.

13 Smith HL, Li W, Cheetham ME: Molecular chaperones and neuronal proteostasis. Semin Cell Dev Biol 2015; 40: 142-152.

14 Magen D, Georgopoulos C, Bross P et al: Mitochondrial hsp60 chaperonopathy causes an autosomal-recessive neurodegenerative disorder linked to brain hypomyelination and leukodystrophy. Am J Hum Genet 2008; 83: 30-42.

15 Hansen JJ, Durr A, Cournu-Rebeix I et al: Hereditary spastic paraplegia SPG13 is associated with a mutation in the gene encoding the mitochondrial chaperonin Hsp60. Am J Hum Genet 2002; 70: 1328-1332.

16 Edvardson S, Cinnamon Y, Ta-Shma A et al: A deleterious mutation in DNAJC6 encoding the neuronal-specific clathrin-uncoating co-chaperone auxilin, is associated with juvenile parkinsonism. PLoS One 2012; 7: e36458.

17 Vilarino-Guell C, Rajput A, Milnerwood AJ et al: DNAJC13 mutations in Parkinson disease. Hum Mol Genet 2014; 23: 1794-1801.

18 Davey KM, Parboosingh JS, McLeod DR et al: Mutation of DNAJC19, a human homologue of yeast inner mitochondrial membrane co-chaperones, causes DCMA syndrome, a novel autosomal recessive Barth syndrome-like condition. J Med Genet 2006; 43: 385-393.

19 Ojala T, Polinati P, Manninen T et al: New mutation of mitochondrial DNAJC19 causing dilated and noncompaction cardiomyopathy, anemia, ataxia, and male genital anomalies. Pediatr Res 2012; 72: 432-437.

Supplementary Information accompanies this paper on European Journal of Human Genetics website (http://www.nature.com/ejhg) 